ఠ

\title{
Are the timings and risk factors changing? Survival analysis of timing of first antenatal care visit among pregnant women in Nigeria (2003-20 I3)
}

This article was published in the following Dove Press journal:

International Journal of Women's Health

30 October 2017

Number of times this article has been viewed

\author{
Adeniyi Francis \\ Fagbamigbe ${ }^{1,2}$ \\ Baitshephi Mashabe' \\ Lornah Lepetu' \\ Clearance Abel' \\ 'Department of Mathematics and \\ Statistical Sciences, Botswana \\ International University of Science \\ and Technology, Palapye, Botswana; \\ ${ }^{2}$ Department of Epidemiology and \\ Medical Statistics, University of \\ Ibadan, Ibadan, Nigeria
}

Background: Child and maternal mortality and morbidity remain among the top global health challenges despite various efforts and multitude of resources directed to improving this situation over time. This study assessed trend of the timings of first antenatal care (ANC) visit in Nigeria and also identified the risk factors associated with it.

Methods: The data obtained from three consecutive Nigerian Demographic and Health Surveys in 2003, 2008, and 2013 were pooled. We focused on the ANC attendance history during the current pregnancies or the last pregnancies within 5 years preceding the survey irrespective of how the pregnancy ended. The gestational age at time of first ANC visit was computed as the survival time, while others who did not attend ANC were censored. Basic descriptive statistics and survival analysis methods were used to analyze the data.

Results: A total of 45,690 pregnancies were studied, of which $70 \%$ were from rural areas. Mothers were mostly (45\%) aged 25-34 years and $47.1 \%$ had no formal education, while only $37.9 \%$ were involved in decisions on the use of health care facility. Prevalence of ANC use was $60.5 \%$ in 2008 and $65.8 \%$ in both 2003 and 2013. Less than one-third (32.3\%) of the women accessed ANC within first 3 months of pregnancy, with highest rate (41.7\%) among women with higher education and those from North Central Nigeria (42.7\%). The hazard of the timing of first ANC visit was higher in years 2003 and 2013 than in 2008.

Conclusion: Initiation of ANC visit in Nigeria is generally late with most women making first visit during second trimester, with significant variations across the years studied. The increase in coverage of ANC recorded in 2003 and 2013 was not accompanied by earlier commencement of ANC visit. Maternal health stakeholders should do more to ensure that all pregnant women start ANC visit earlier.

Keywords: antenatal care, timing of first ANC visit, Nigeria, women autonomy, maternal health

\section{Introduction}

Maternal and new born mortality and morbidity remain among the top global health challenges despite various efforts and huge resources directed to improving this situation over time. ${ }^{1}$ Majority of child and maternal deaths can be prevented if women have access to life-saving treatments, well-equipped hospitals, and trained birth attendants. One of the core strategies of the United Nation's Sustainable Development Goal is aimed at improving maternal health and reducing maternal mortality ratio by threequarters. ${ }^{2}$ The goal was to enable women to have access to high-quality maternal health services. One way to curve down maternal and perinatal morbidity and mortality is through the use of antenatal care (ANC) service. , $^{3,4}$
Correspondence: Baitshephi Mashabe Department of Mathematics and Statistical Sciences, Botswana International University of Science and Technology, Private Bag 016, Palapye, Botswana

Tel +267 72960349

Email mashabeb@biust.ac.bw 
ANC is one of the four pillars of safe motherhood. ${ }^{5}$ ANC is a medical service provided to a woman throughout her pregnancy to ensure that pregnancy and childbirth is a success. ${ }^{6}$ With improved understanding of the need for women to prepare physically, mentally, and even logistically for childbirth, ANC is recognized as a key maternal service that aids in improving a wide range of health outcomes for women and children..$^{7-9}$ It gives opportunity for early detection of possible risk factors and provides opportunity for a full range of health promoting services. ${ }^{10}$ ANC presents an opportunity to deliver interventions for improving maternal nutrition, providing health education, and encouraging skilled attendance at birth and use of facilities for emergency obstetric care. ${ }^{11,12}$ Opportunities for interventions may include services such as weight, diabetes, and high blood pressure management; immunization against tetanus; treatment of malaria; and HIV testing and prevention of mother to child transmission..$^{10}$ It also facilitates the early detection and management or referral of pregnancy-related complications. ${ }^{5}$ Increased access and use of better quality health care during pregnancy and childbirth can prevent pregnancy-related deaths and diseases, as well as improve women and adolescent girl's experience of pregnancy and childbirth.

Women are expected to visit ANC service at least four times at specified intervals during pregnancy. ${ }^{13}$ Although substantial progress has been made over the past two decades in many parts of the world, such successes are minimal in developing countries. ${ }^{4,14}$ Globally, only $64 \%$ of women receive ANC four or more times throughout their pregnancy. Reports showed slower progress in Sub-Saharan Africa than in other regions, at a coverage rate of only $4 \%$ during the past decade. Currently, $71 \%$ of women worldwide receive any (at least one visit) ANC; in industrialized countries, over $95 \%$ of pregnant women have access to ANC. In Sub-Saharan Africa, $69 \%$ of pregnant women have at least one ANC visit, more than in South Asia at 54\%. ${ }^{4}$ The rate in Nigeria is lower than the global average. Only about two-thirds of pregnant women accessed ANC as of 2013, while just over a half would make the recommended four visits. ${ }^{15-17}$

Different factors influence the use of ANC. Literature has unanimously identified maternal age, education, parity, income, standard of living of households, cultural barriers, and distance to ANC provider as some of the factors affecting ANC utilization. ${ }^{14,18-21}$ Several states in Nigeria have begun to offer free maternal health services in response to poor maternal health care. ${ }^{21}$ In 2007, the Northern Governors' Forum Summit pledged to end preventable maternal deaths, most notably in Northern Nigeria. ${ }^{22}$
While it is necessary to have at least four ANC visits, timing of the first ANC visit is equally important for every pregnant woman; ${ }^{12,22}$ this is primarily due to the fact that different services and interventions are available for different gestational ages. More so, for many of the essential interventions in ANC, it is crucial to have early identification of the underlying conditions. For example, precautions for the prevention of congenital syphilis, control of anemia, and prevention of malarial complications should be taken on time. ${ }^{4}$ Hence, the first ANC visit should be as early as possible in pregnancy, preferably in the first trimester, and the last visit should be at around 37 weeks or near the expected date of birth to ensure that appropriate advice and care have been provided to prevent and manage problems such as multiple births (eg, twins), post-maturity births (eg, birth after 42 weeks of pregnancy, which carries an increased risk of fatal death), and abnormal positions of the baby (eg, breech, where the baby's head is not the presenting part at birth). ${ }^{4}$

Considering the marginal increase in ANC utilization reported in Nigeria over time, ${ }^{15,23}$ we designed this study to investigate if the increase has been met with changes in timing of first ANC over the years. Our objective was to assess trend of the timings of first ANC visit among pregnant women in Nigeria using three waves of nationally representative data. The current study also identified the different risk factors associated with the first timing of ANC visit in Nigeria.

Until now, available studies on the timing of first ANC visit in Nigeria have compared proportions of first visits in each trimester without emphasis on progression of the times and total exclusion of women who had not made any visit irrespective of the gestational age at data collection time. The current study accommodated currently pregnant women and monitored their uptake of ANC services alongside women who had already delivered their child. An up-to-date information of the rate at which pregnant women uptake over the years will assist maternal health programmers, policy makers, as well as other stakeholders in refocusing their interventions to ensure timely and optimal uptake of ANC services.

\section{Methods}

\section{Study setting and source of data}

In this study, we pooled the data obtained from three consecutive surveys conducted by Nigerian Demographic and Health Survey (NDHS) in 2003, 2008, and 2013. NDHS is the Nigerian episode of the global demographic and health surveys regularly conducted among women aged 15-49 years in some developing countries by ICF Macro, USA. ${ }^{15,23}$ The surveys were cross-sectional, nationally representative, and 
engaged multistage approach in sampling the respondents and collected information about household and reproductive health of the participants. The stages included selection of local government area, selection of clusters (enumeration areas), selection of households, and selection of individual respondents from the households. We were particularly interested in the timing of first ANC visit to the providers. To achieve this, we focused on the ANC attendance history during the current pregnancies or the last pregnancies within 5 years preceding the survey by respondents who were not currently pregnant irrespective of how the pregnancy ended. Written informed consent for participation in the study was obtained from all the participants aged 15-49 years and additional written consent were obtained from parents and guardians of participants who had not attained 18 years of age.

Among the 7,620, 33,385, and 38,948 women who participated in the 2003, 2008, and 2013 surveys, respectively, only 4,167 (54.7\%), 19,340 (57.9\%), and 22,183 (57.0\%) pregnancies, respectively, totaling 45,690 pregnancies met the inclusion criteria of our study. All analysis in this study was therefore based on the history of ANC attendance of women during their last or current pregnancy.

\section{Data extraction}

We extracted self-reported women background characteristics, and sexual and reproductive history. All women who had either at least one child during the 5 years preceding the survey or currently pregnant were included in the analysis. We computed the survival time as the gestational age at the time of first ANC visit. However, the pregnancy could have been lost to follow-up, delivered, terminated, or aborted without accessing ANC or could be that the women were currently pregnant and had not visited ANC as of the time of the survey. For both cases, the survival time is censored and computed as the age when the pregnancy was last seen, delivered, aborted, or terminated or the gestational age at the time of the survey.

\section{Variables}

The outcome of interest in this study is the timing of first ANC visits expressed in months. The independent women characteristics used in this study and categorizations are women age (15-19, 20-24, 25-34, 35+ years), highest education (none, primary, secondary, higher), marital status (never married, currently married, formerly married), religion (Islam, Christian/Catholic, others), tribe (Hausa/ Fulani, Yoruba, Igbo, others), women involvement in the spending of her earnings (Yes, No), women participation in decisions on her use of health care facility (Yes, No), whether the pregnancy was desired as of then or not (wanted then, not wanted then), number of previous pregnancies (none, 1 , 2-3, 4-6, 7+); whether mother was currently working or not (Yes, No), household wealth (poorest, middle, richest); region of residence (North Central, North East, North West, South East, South South; South West), residence (urban, rural), whether distance to health facilities is a big problem or not (big problem, not a big problem). Our choice of explanatory variables was based on identified risk factors of ANC utilization in literature. ${ }^{14,24-27}$

\section{Data analysis}

Basic descriptive statistics were engaged to show the distribution of the studied women (Table 1), and the prevalence of ANC utilization and proportion who started ANC visits during first trimester by women characteristics is summarized in Table 2. Survival analysis methods were used to estimate the incidence rate (IR), determine the medial survival time, and to model the determinants of timing of first ANC visits (Table 3). The adjusted hazard ratios (aHRs) are reported in Table 4. Stata version 14 was used for all analyses. Prior to analysis, sampling weights provided by DHS were applied to the data so as to make provisions for unequal sample sizes across groups. Survey design and sampling errors were adjusted for in all the analyses. Statistical significance was determined at $p$-value $=0.05$. Ethical clearance was obtained earlier by the National Population Commission and ICF Macro from Nigeria National Health Research Ethics Committee as reported earlier. ${ }^{15,23}$

In the current study, the population at risk are pregnant women in the data, since pregnant women are exposed to the "risk" of visiting ANC facility during their course of their pregnancy, while the end point of interest is uptake of ANC.

We evaluated the survivor function $S(t)$ and hazard function $h(t)$ of the timing of first ANC visit in this study. We defined the survivor function as the probability that a pregnant woman has not accessed ANC as of specified time $t$. It is the probability of not "failing" before time $t$, whereas the hazard function is the probability that a pregnant woman would access ANC after time $t$, given that she had not visited ANC at time $t$.

The Kaplan-Meier estimates of $S(t)$ were obtained and used to plot the respective survival functions. Log-rank tests were used to test whether the survival curves for different categories of variables studied were significant or not. 
Table I Distribution of the pregnant women studied, by characteristics and the DHS year

\begin{tabular}{|c|c|c|c|c|c|}
\hline \multirow[t]{2}{*}{ Characteristics } & \multicolumn{2}{|c|}{ Total $=45,690$} & \multirow{2}{*}{$\frac{2003}{4,167}$} & \multirow{2}{*}{$\frac{2008}{19,340}$} & \multirow{2}{*}{$\frac{2013}{22,183}$} \\
\hline & $\mathbf{n}$ & $\%$ & & & \\
\hline \multicolumn{6}{|l|}{ Age, years } \\
\hline $15-19$ & 3,777 & 8.3 & 9.7 & 8.3 & 7.8 \\
\hline $20-24$ & 9,018 & 19.7 & 20.7 & 19.7 & 19.6 \\
\hline $25-34$ & 20,667 & 45.2 & 44.9 & 45.3 & 45.3 \\
\hline $35-49$ & 12,229 & 26.8 & 24.7 & 26.7 & 27.3 \\
\hline \multicolumn{6}{|l|}{ Mother education } \\
\hline No education & 21,502 & 47.1 & 48.5 & 47.6 & 45.7 \\
\hline Primary & 9,857 & 21.6 & 23.4 & 22.3 & 19.9 \\
\hline Secondary & 11,577 & 25.3 & 23.8 & 24.2 & 27.7 \\
\hline Higher & 2,755 & 6.0 & 4.4 & 5.8 & 6.8 \\
\hline \multicolumn{6}{|l|}{ Marital status } \\
\hline Never & I,582 & 3.5 & 3.9 & 3.4 & 3.4 \\
\hline Married/LWSP & 42,666 & 93.4 & 92.1 & 93.6 & 93.4 \\
\hline Formerly married & $\mathrm{I}, 44 \mathrm{I}$ & 3.2 & 4.1 & 3.0 & 3.1 \\
\hline \multicolumn{6}{|l|}{ Religion } \\
\hline Islam & 23,798 & 52.1 & 15.2 & 54.2 & 57.9 \\
\hline Xtian & 18,679 & 40.9 & 24.5 & 43.4 & 40.7 \\
\hline Others & 3,213 & 7.0 & 60.4 & 2.5 & 1.4 \\
\hline \multicolumn{6}{|l|}{ Tribe } \\
\hline Hausa/Fulani & 16,772 & 36.7 & 30.0 & 36.0 & 39.7 \\
\hline Yoruba & 5,391 & 11.8 & 11.3 & 12.0 & 11.6 \\
\hline Igbo & $4,7 \mid 4$ & 10.3 & 12.0 & 10.3 & 9.9 \\
\hline Others & 18,813 & 41.2 & 46.7 & 41.7 & 38.9 \\
\hline \multicolumn{6}{|c|}{ Involved in her income spending } \\
\hline Yes & 22,093 & 86.9 & 90.6 & 84.3 & 89.9 \\
\hline No & 3,332 & 13.1 & 9.5 & 15.7 & 10.1 \\
\hline \multicolumn{6}{|c|}{ Involved in her health care decision } \\
\hline Yes & 16,248 & 37.9 & 23.6 & 40.7 & 36.8 \\
\hline No & 26,679 & 62.2 & 76.4 & 59.3 & 63.2 \\
\hline \multicolumn{6}{|l|}{ Residence } \\
\hline Urban & 13,679 & 29.9 & 35.4 & 27.1 & 33.6 \\
\hline Rural & 32,011 & 70.1 & 64.6 & 73.0 & 66.4 \\
\hline \multicolumn{6}{|l|}{ Zone } \\
\hline North Central & 7,700 & 16.9 & 16.5 & 18.1 & 14.8 \\
\hline North East & 9,685 & 21.2 & 22.4 & 21.8 & 19.8 \\
\hline North West & 13,073 & 28.6 & 30.0 & 26.5 & 32.0 \\
\hline South East & 3,774 & 8.3 & 8.1 & 8.4 & 8.1 \\
\hline South South & 5,661 & 12.4 & II.I & 12.5 & 12.6 \\
\hline South West & 5,797 & 12.7 & 11.9 & 12.8 & 12.7 \\
\hline \multicolumn{6}{|c|}{ Distance to health facility is a problem } \\
\hline Big problem & 16,476 & 36.2 & 25.6 & 40.1 & 32.2 \\
\hline No problem & 29,035 & 63.8 & 74.4 & 60.0 & 67.8 \\
\hline \multicolumn{6}{|l|}{ Working } \\
\hline Yes & 31,180 & 68.5 & 65.0 & 68.0 & 70.5 \\
\hline No & $|4,3| \mid$ & 31.5 & 35.0 & 32.0 & 29.5 \\
\hline \multicolumn{6}{|l|}{ Wealth quintile } \\
\hline Poorest & 21,425 & 46.9 & 42.5 & 48.8 & 44.7 \\
\hline Middle & 8,985 & 19.7 & 20.1 & 19.5 & 19.9 \\
\hline Richest & 15,280 & 33.4 & 37.4 & 31.8 & 35.4 \\
\hline \multicolumn{6}{|l|}{ Wanted the pregnancy } \\
\hline Wanted then & 39,191 & 89.4 & 84.8 & 89.5 & 90.4 \\
\hline Wanted later & 2,899 & 6.6 & 9.5 & 5.7 & 7.6 \\
\hline Not at all & $\mathrm{I}, 746$ & 4.0 & 5.7 & 4.8 & 2.1 \\
\hline
\end{tabular}

Table I (Continued)

\begin{tabular}{|c|c|c|c|c|c|}
\hline \multirow[t]{2}{*}{ Characteristics } & \multicolumn{2}{|c|}{ Total $=45,690$} & \multirow{2}{*}{$\frac{2003}{4,167}$} & \multirow{2}{*}{$\frac{2008}{19,340}$} & \multirow{2}{*}{$\frac{2013}{22,183}$} \\
\hline & $\mathbf{n}$ & $\%$ & & & \\
\hline \multicolumn{6}{|c|}{ Number of previous pregnancies } \\
\hline None & 8,540 & 17.4 & 18.6 & 17.1 & 17.5 \\
\hline I & $7,|7|$ & 16.0 & 15.5 & 16.2 & 15.7 \\
\hline $2-3$ & 12,384 & 27.5 & 26.2 & 27.5 & 27.9 \\
\hline 4-6 & 11,640 & 25.9 & 25.3 & 25.9 & 26.1 \\
\hline $7+$ & 5,957 & 13.3 & 14.5 & 13.3 & 12.8 \\
\hline Total & 45,690 & 100 & 9.1 & 42.3 & 48.6 \\
\hline
\end{tabular}

Abbreviations: LWSP, living with sexual partner; DHS, Demographic and Health Survey.

We computed the incidence rate (IR) for each survey year, as well as for all the explanatory variables and overall IR of accessing ANC. The IR is the probability that an event will occur at time $t_{k+1}$ given that it did not occur at time $t_{k}$. In this study, IR is the probability of uptaking ANC at a time (month) provided that the pregnant women have not attended ANC at that time.

Cox-proportional hazard regression model was used to model the determinants of the timing of first ANC visit. We determined Cox regression estimates for all levels of each of the covariates. The HR $>1$ implies higher susceptibility to earlier uptake of ANC, HR $<1$ suggests lower susceptibility, while $\mathrm{HR}=1$ indicates no difference in ANC uptake timings. We built $95 \%$ confidence interval around each of the HRs to further establish their significance. This procedure has been used in previous studies. ${ }^{28,29}$

We determined the significance of each risk factor in independent bivariate Cox regression models before controlling for them and estimating the unadjusted HR in multiple Cox regression model. This was done for each of the survey years and then collectively.

\section{Definition of technical terms}

Survival time: this is the censored gestational age at the start of ANC.

Incidence rate: the probability of developing the event of interest per unit measurement time. In our study, it is the probability of accessing ANC within 1 month.

Median survival time: this is a statistic that describes the time before half of the subjects would have developed the event of interest in a censored data. In our study, it is the number of months before half of the women started ANC.

Hazard ratio: this is the ratio that describes the likelihood of a particular group developing the event of interest relative to another group. 
Table 2 Prevalence of ANC utilization and proportion who started ANC visits during first trimester by women characteristics

\begin{tabular}{|c|c|c|c|c|c|c|c|c|}
\hline \multirow[t]{2}{*}{ Characteristics } & \multicolumn{4}{|c|}{$\%$ who accessed ANC } & \multicolumn{4}{|c|}{$\%$ of users starting ANC in first trimester } \\
\hline & 2003 & 2008 & 2013 & All & 2003 & 2008 & 2013 & All \\
\hline \multicolumn{9}{|l|}{ Age, years } \\
\hline $15-19$ & 53.7 & 44.9 & 54.1 & $48.6^{*}$ & 26.9 & 29.2 & 26.5 & 28.0 \\
\hline $20-24$ & 62.0 & 56.7 & 63.2 & $59.3^{*}$ & 30.3 & 33.9 & 33.2 & $33.3^{*}$ \\
\hline $25-34$ & 68.6 & 64.7 & 68.0 & $66.1^{*}$ & 33.7 & 33.3 & 33.5 & 33.4 \\
\hline $35-49$ & 67.8 & 59.9 & 65.7 & $62.4^{*}$ & 34.9 & 29.7 & 30.9 & $30.5^{*}$ \\
\hline \multicolumn{9}{|l|}{ Mother education } \\
\hline No education & 43.0 & 34.6 & 42.1 & $37.7^{*}$ & 30.4 & 30.1 & 26.7 & $28.9 *$ \\
\hline Primary & 82.2 & 76.4 & 77.3 & $77.2^{*}$ & 31.2 & 30.4 & 31.7 & $30.9 *$ \\
\hline Secondary & 93.7 & 90.6 & 89.8 & $90.5^{*}$ & 35.8 & 33.3 & 34.7 & $34.0 *$ \\
\hline Higher & 98.7 & 98.3 & 98.8 & 98.5 & 40.0 & 41.3 & 42.7 & 41.7 \\
\hline \multicolumn{9}{|l|}{ Marital status } \\
\hline Never & 86.7 & 75.4 & 78.9 & 77.5 & 37.8 & 25.2 & 28.6 & $27.6^{*}$ \\
\hline Married/LWSP & 65.0 & 59.9 & 64.9 & $62.0 *$ & 33.1 & 32.5 & 32.5 & $32.6 *$ \\
\hline Formerly married & 72.6 & 67.8 & 73.4 & 70.2 & 24.0 & 31.9 & 31.2 & 30.8 \\
\hline \multicolumn{9}{|l|}{ Religion } \\
\hline Islam & 89.6 & 43.6 & 53.8 & $48.4^{*}$ & 39.0 & 29.5 & 28.2 & $29.4^{*}$ \\
\hline Xtian & 88.4 & 83.3 & 83.4 & $83.6 *$ & 34.4 & 34.2 & 36.4 & $34.9 *$ \\
\hline Others & 51.8 & 46.9 & 51.9 & 50.8 & 29.4 & 29.7 & 30.9 & 29.6 \\
\hline \multicolumn{9}{|l|}{ Tribe } \\
\hline Hausa/Fulani & 43.6 & 31.6 & 44.5 & $37.1^{*}$ & 25.9 & 26.4 & 22.4 & $24.6^{*}$ \\
\hline Yoruba & 96.7 & 94.9 & 97.2 & $95.8^{*}$ & 41.4 & 29.6 & 35.8 & $32.6 *$ \\
\hline Igbo & 95.7 & 91.1 & 95.7 & $96.0^{*}$ & 34.8 & 35.6 & 37.8 & $36.3^{*}$ \\
\hline Others & 66.2 & 68.6 & 70.6 & $69.0^{*}$ & 32.3 & 34.6 & 35.7 & $34.7^{*}$ \\
\hline \multicolumn{9}{|c|}{ Involved in her income spending } \\
\hline Yes & 69.6 & 65.4 & 69.1 & $67.2^{*}$ & 34.9 & 32.4 & 32.6 & $32.7 *$ \\
\hline No & 80.2 & 60.7 & 71.1 & $64.9 *$ & 37.0 & 38.5 & 32.5 & $36.5^{*}$ \\
\hline \multicolumn{9}{|c|}{ Involved in her health care decision } \\
\hline Yes & 81.6 & 76.8 & 80.6 & $78.3^{*}$ & 37.8 & 32.6 & 36.4 & $34.1 *$ \\
\hline No & 61.1 & 48.3 & 56.0 & $52.3^{*}$ & 30.8 & 32.3 & 29.4 & $31.1 *$ \\
\hline \multicolumn{9}{|l|}{ Residence } \\
\hline Urban & 85.0 & 86.1 & 88.9 & $87.1^{*}$ & 33.5 & 31.0 & 30.8 & $31.2 *$ \\
\hline Rural & 55.6 & 51.2 & 54.0 & $52.4^{*}$ & 32.4 & 33.0 & 33.6 & $33.2 *$ \\
\hline \multicolumn{9}{|l|}{ Zone } \\
\hline North Central & 75.2 & 73.8 & 78.8 & $75.4^{*}$ & 45.3 & 41.2 & 45.0 & $42.7^{*}$ \\
\hline North East & 53.2 & 48.6 & 61.1 & $52.9 *$ & 29.0 & 32.1 & 32.0 & $31.8^{*}$ \\
\hline North West & 44.1 & 27.5 & 42.2 & $34.4^{*}$ & 20.5 & 23.2 & 19.5 & $21.2 *$ \\
\hline South East & 98.2 & 90.3 & 95.6 & $92.7^{*}$ & 33.4 & 34.9 & 37.8 & $35.7^{*}$ \\
\hline South South & 85.1 & 80.4 & 70.9 & $77.5^{*}$ & 33.9 & 32.1 & 28.8 & $31.2 *$ \\
\hline South West & 97.6 & 94.5 & 92.5 & $94.1^{*}$ & 37.3 & 26.5 & 34.3 & $29.9 *$ \\
\hline \multicolumn{9}{|c|}{ Distance to health facility is a problem } \\
\hline Big problem & 49.2 & 50.6 & 46.4 & $49.3 *$ & 31.3 & 32.3 & 29.6 & $31.5 *$ \\
\hline No problem & 71.7 & 67.2 & 74.8 & $70.3^{*}$ & 33.2 & 32.2 & 33.2 & $32.7^{*}$ \\
\hline \multicolumn{9}{|l|}{ Working } \\
\hline Yes & 71.4 & 65.4 & 69.9 & $67.4^{*}$ & 34.8 & 33.0 & 33.0 & $33.1 *$ \\
\hline No & 55.3 & 49.8 & 54.9 & $51.9 *$ & 28.2 & 30.1 & 30.3 & 30.0 \\
\hline \multicolumn{9}{|l|}{ Wealth quintile } \\
\hline Poorest & 45.6 & 37.4 & 42.0 & $39.5^{*}$ & 31.3 & 30.9 & 30.6 & 30.8 \\
\hline Middle & 66.6 & 71.3 & 74.5 & $72.0^{*}$ & 31.2 & 32.4 & 32.9 & $32.4^{*}$ \\
\hline Richest & 90.4 & 90.8 & 91.5 & 91.0 & 34.6 & 33.1 & 33.3 & $33.3^{*}$ \\
\hline \multicolumn{9}{|l|}{ Wanted the pregnancy } \\
\hline Wanted then & 63.6 & 59.3 & 64.1 & $61.3^{*}$ & 32.9 & 32.5 & 32.3 & $32.5^{*}$ \\
\hline Wanted later & 75.5 & 70.0 & 79.8 & $74.4^{*}$ & 34.0 & 33.7 & 34.0 & 33.8 \\
\hline Not at all & 83.3 & 73.6 & 80.9 & $76.0^{*}$ & 31.5 & 26.9 & 28.2 & 27.7 \\
\hline
\end{tabular}


Table 2 (Continued)

\begin{tabular}{|c|c|c|c|c|c|c|c|c|}
\hline \multirow[t]{2}{*}{ Characteristics } & \multicolumn{4}{|c|}{$\%$ who accessed ANC } & \multicolumn{4}{|c|}{$\%$ of users starting ANC in first trimester } \\
\hline & 2003 & 2008 & 2013 & All & 2003 & 2008 & 2013 & All \\
\hline \multicolumn{9}{|c|}{ Number of previous pregnancies } \\
\hline None & 72.3 & 66.5 & 72.7 & $69.1 *$ & 36.0 & 32.0 & 33.2 & $32.8 *$ \\
\hline 1 & 69.6 & 65.2 & 70.7 & $67.3^{*}$ & 34.7 & 38.1 & 37.1 & 37.5 \\
\hline $2-3$ & 68.0 & 63.9 & 68.4 & $65.7^{*}$ & 33.2 & 31.7 & 33.3 & $32.4^{*}$ \\
\hline 4-6 & 62.3 & 58.5 & 62.7 & $60.2^{*}$ & 30.1 & 30.4 & 30.1 & $30.3^{*}$ \\
\hline $7+$ & 57.6 & 45.5 & 51.1 & $48.4^{*}$ & 31.0 & 28.4 & 26.0 & 27.9 \\
\hline Total & 65.8 & 60.5 & 65.5 & $62.6 *$ & 32.9 & 32.2 & 32.4 & $32.3^{*}$ \\
\hline
\end{tabular}

Note: *Significantly different across DHS years at $5 \%$.

Abbreviations: ANC, antenatal care; DHS, Demographic and Health Survey; LWSP, living with sexual partner.

Table 3 The incidence rate, median survival time, and log-rank test of timing of first ANC visit by selected characteristics and survey years

\begin{tabular}{|c|c|c|c|c|c|c|c|c|}
\hline \multirow[t]{2}{*}{ Characteristics } & \multicolumn{4}{|c|}{ Incidence rate } & \multicolumn{4}{|c|}{ Median survival time (months) } \\
\hline & 2003 & 2008 & 2013 & All & 2003 & 2008 & 2013 & All \\
\hline \multicolumn{9}{|l|}{ Age, years } \\
\hline $15-19$ & 0.07 & 0.06 & 0.07 & 0.06 & 9 & a & a & $\mathrm{a}$ \\
\hline $20-24$ & 0.10 & 0.09 & 0.11 & 0.10 & 6 & 6 & 6 & 6* \\
\hline $25-34$ & 0.12 & 0.11 & 0.12 & 0.12 & 5 & 6 & 5 & 5 \\
\hline $35-49$ & 0.11 & 0.09 & 0.11 & 0.10 & 6 & 6 & 6 & 6 \\
\hline \multicolumn{9}{|l|}{ Mother education } \\
\hline No education & 0.06 & 0.05 & 0.06 & 0.06 & $\mathrm{a}$ & $\mathrm{a}$ & $\mathrm{a}$ & $\mathrm{a}$ \\
\hline Primary & 0.15 & 0.13 & 0.14 & 0.14 & 5 & 5 & 5 & $5^{*}$ \\
\hline Secondary & 0.18 & 0.17 & 0.17 & 0.17 & 4 & 5 & 5 & 5 \\
\hline Higher & 0.20 & 0.21 & 0.21 & 0.21 & 4 & 4 & 4 & 4 \\
\hline \multicolumn{9}{|l|}{ Marital status } \\
\hline Never & 0.12 & 0.10 & 0.11 & 0.11 & 6 & 6 & 6 & $6 *$ \\
\hline Formerly married & 0.11 & 0.10 & 0.11 & 0.10 & 6 & 6 & 6 & 6 \\
\hline Married/LWSP & 0.12 & 0.11 & 0.13 & 0.12 & 6 & 6 & 5 & 5 \\
\hline \multicolumn{9}{|l|}{ Religion } \\
\hline Islam & 0.17 & 0.06 & 0.08 & 0.08 & 5 & $\mathrm{a}$ & 7 & $8^{*}$ \\
\hline Xtian & 0.16 & 0.15 & 0.16 & 0.16 & 5 & 5 & 5 & 5 \\
\hline Others & 0.08 & 0.07 & 0.08 & 0.08 & 7 & $\mathrm{a}$ & 7 & 7 \\
\hline \multicolumn{9}{|l|}{ Tribe } \\
\hline Hausa/Fulani & 0.06 & 0.04 & 0.06 & 0.05 & a & a & a & $\mathrm{a}$ \\
\hline Yoruba & 0.21 & 0.19 & 0.21 & 0.20 & 4 & 5 & 4 & $4^{*}$ \\
\hline lgbo & 0.18 & 0.18 & 0.20 & 0.19 & 5 & 5 & 4 & 4 \\
\hline Others & 0.11 & 0.12 & 0.12 & 0.12 & 6 & 5 & 5 & 5 \\
\hline \multicolumn{9}{|c|}{ Involved in her income spending } \\
\hline Yes & 0.12 & 0.11 & 0.12 & 0.12 & 5 & 6 & 5 & 5 \\
\hline No & 0.14 & 0.10 & 0.12 & 0.11 & 5 & 6 & 5 & 5 \\
\hline \multicolumn{9}{|c|}{ Involved in her health care decision } \\
\hline Yes & 0.15 & 0.14 & 0.15 & 0.15 & 5 & 5 & 5 & $5^{*}$ \\
\hline No & 0.10 & 0.07 & 0.09 & 0.08 & 6 & a & 7 & 7 \\
\hline \multicolumn{9}{|l|}{ Residence } \\
\hline Urban & 0.16 & 0.17 & 0.17 & 0.17 & 5 & 5 & 5 & $5^{*}$ \\
\hline Rural & 0.09 & 0.08 & 0.09 & 0.08 & 7 & 8 & 7 & 7 \\
\hline \multicolumn{9}{|l|}{ Zone } \\
\hline North Central & 0.14 & 0.13 & 0.15 & 0.14 & 5 & 5 & 5 & $5^{*}$ \\
\hline North East & 0.08 & 0.07 & 0.10 & 0.09 & 7 & $\mathrm{a}$ & 6 & 7 \\
\hline North West & 0.06 & 0.04 & 0.06 & 0.05 & & & & \\
\hline South East & 0.19 & 0.17 & 0.20 & 0.19 & 5 & 5 & 4 & 4 \\
\hline South South & 0.14 & 0.14 & 0.12 & 0.13 & 5 & 5 & 6 & 5 \\
\hline South West & 0.20 & 0.19 & 0.19 & 0.19 & 4 & 5 & 4 & 5 \\
\hline
\end{tabular}


Table 3 (Continued)

\begin{tabular}{|c|c|c|c|c|c|c|c|c|}
\hline \multirow[t]{2}{*}{ Characteristics } & \multicolumn{4}{|c|}{ Incidence rate } & \multicolumn{4}{|c|}{ Median survival time (months) } \\
\hline & 2003 & 2008 & 2013 & All & 2003 & 2008 & 2013 & All \\
\hline \multicolumn{9}{|c|}{ Distance to health facility is big problem } \\
\hline Big problem & 0.07 & 0.08 & 0.07 & 0.07 & a & 8 & a & a \\
\hline No problem & 0.12 & 0.11 & 0.13 & 0.12 & 5 & 5 & 5 & 5 \\
\hline \multicolumn{9}{|l|}{ Working } \\
\hline Yes & 0.13 & 0.11 & 0.12 & 0.12 & 5 & 6 & 5 & $5^{*}$ \\
\hline No & 0.08 & 0.07 & 0.09 & 0.08 & 7 & a & 7 & 7 \\
\hline \multicolumn{9}{|l|}{ Wealth quintile } \\
\hline Poorest & 0.07 & 0.05 & 0.06 & 0.06 & a & $\mathrm{a}$ & $\mathrm{a}$ & $\mathrm{a}$ \\
\hline Middle & 0.11 & 0.12 & 0.13 & 0.12 & 6 & 5 & 5 & $5^{*}$ \\
\hline Richest & 0.17 & 0.18 & 0.18 & 0.18 & 5 & 5 & 4 & 5 \\
\hline \multicolumn{9}{|c|}{ Wanted the pregnancy } \\
\hline Wanted then & 0.11 & 0.10 & 0.11 & 0.10 & 6 & 6 & 6 & $6^{*}$ \\
\hline Wanted later & 0.13 & 0.12 & 0.15 & 0.14 & 5 & 5 & 5 & 5 \\
\hline Not at all & 0.14 & 0.13 & 0.15 & 0.13 & 5 & 5 & 5 & 5 \\
\hline \multicolumn{9}{|c|}{ Previous pregnancies } \\
\hline None & 0.10 & 0.09 & 0.11 & 0.09 & 6 & 7 & 6 & $6^{*}$ \\
\hline 1 & 0.13 & 0.11 & 0.13 & 0.12 & 5 & 5 & 5 & 5 \\
\hline $2-3$ & 0.12 & 0.11 & 0.12 & 0.12 & 5 & 6 & 5 & 5 \\
\hline $4-6$ & 0.11 & 0.09 & 0.11 & 0.10 & 6 & 6 & 6 & 6 \\
\hline $7+$ & 0.09 & 0.07 & 0.08 & 0.08 & 6 & $\mathrm{a}$ & 7 & 8 \\
\hline Total & 0.11 & 0.10 & 0.11 & 0.10 & 6 & 6 & 6 & 6 \\
\hline
\end{tabular}

Notes: a: median survival time cannot be computed. *Significantly different at $5 \%$ log-rank test.

Abbreviations: ANC, antenatal care; LWSP, living with sexual partner.

Table 4 The adjusted risk factors of timing of first ANC visit by selected characteristics and survey years

\begin{tabular}{|c|c|c|c|c|}
\hline \multirow[t]{2}{*}{ Characteristics } & \multicolumn{4}{|c|}{ Adjusted hazard ratios $(95 \% \mathrm{Cl})$} \\
\hline & 2003 & 2008 & 2013 & All women \\
\hline \multicolumn{5}{|l|}{ DHS } \\
\hline 2008 & & & & Reference \\
\hline 2003 & & & & $1.19(1.12-1.26)$ \\
\hline 2013 & & & & $1.08(1.06-1.11)$ \\
\hline \multicolumn{5}{|l|}{ Age, years } \\
\hline $15-19$ & Reference & & & \\
\hline $20-24$ & $1.06(0.88-1.27)$ & $1.16(1.04-1.29)$ & $1.19(1.08-1.31)$ & $1.16(1.09-1.24)$ \\
\hline $25-34$ & $1.18(0.97-1.44)$ & $1.18(1.06-1.31)$ & $1.21(1.10-1.34)$ & $1.20(1.12-1.29)$ \\
\hline $35-49$ & $1.18(0.94-1.48)$ & $1.15(1.01-1.30)$ & $1.24(1.11-1.38)$ & $1.19(1.11-1.29)$ \\
\hline \multicolumn{5}{|l|}{ Mother education } \\
\hline No education & Reference & & & \\
\hline Primary & $1.61(1.43-1.81)$ & $1.72(1.62-1.83)$ & $1.61(1.52-1.70)$ & $1.67(1.6-1.74)$ \\
\hline Secondary & $1.71(1.49-1.95)$ & $1.86(1.74-2.00)$ & $1.76(1.65-1.87)$ & $1.82(1.74-1.9)$ \\
\hline Higher & $1.75(1.42-2.15)$ & $2.04(1.86-2.25)$ & $1.97(1.81-2.14)$ & $1.99(1.87-2.11)$ \\
\hline \multicolumn{5}{|l|}{ Marital status } \\
\hline Never & Reference & & & \\
\hline Formerly married & $1.00(0.78-1.28)$ & $1.10(0.99-1.22)$ & $1.04(0.94-1.15)$ & $1.02(0.82-1.27)$ \\
\hline Married/LWSP & $0.90(0.74-1.10)$ & $1.14(1.03-1.27)$ & $1.15(1.05-1.26)$ & $0.89(0.74-1.09)$ \\
\hline \multicolumn{5}{|l|}{ Religion } \\
\hline Islam & Reference & & & \\
\hline Xtian & $1.02(0.89-1.16)$ & $1.09(1.03-1.16)$ & $0.99(0.94-1.05)$ & $1.02(0.99-1.06)$ \\
\hline Others & $0.82(0.72-0.95)$ & $0.94(0.8 \mathrm{I}-1.09)$ & $0.80(0.67-0.94)$ & $0.84(0.79-0.90)$ \\
\hline \multicolumn{5}{|l|}{ Tribe } \\
\hline Hausa/Fulani & Reference & & & \\
\hline Yoruba & I.I7 (0.94-I.47) & $1.21(1.08-1.35)$ & $1.19(1.08-1.31)$ & $1.19(1.11-1.27)$ \\
\hline lgbo & I.I $(0.92-1.5)$ & $1.27(1.11-1.45)$ & $1.25(1.11-1.40)$ & $1.25(1.15-1.36)$ \\
\hline Others & $1.23(1.06-1.4 I)$ & $1.26(1.16-1.36)$ & $1.14(1.06-1.22)$ & $1.19(1.14-1.25)$ \\
\hline
\end{tabular}


Table 4 (Continued)

\begin{tabular}{|c|c|c|c|c|}
\hline \multirow[t]{2}{*}{ Characteristics } & \multicolumn{4}{|c|}{ Adjusted hazard ratios $(95 \% \mathrm{Cl})$} \\
\hline & 2003 & 2008 & 2013 & All women \\
\hline \multicolumn{5}{|c|}{ Involved in her income spending decision } \\
\hline & Reference & & & \\
\hline Yes & $1.00(0.84-1.18)$ & $1.09(1.01-1.18)$ & $1.06(0.98-1.14)$ & $1.07(1.02-1.13)$ \\
\hline \multicolumn{5}{|l|}{ No } \\
\hline \multicolumn{5}{|c|}{ Involved in her health care decision } \\
\hline Yes & $1.07(0.97-1.18)$ & $1.08(1.03-1.12)$ & $1.02(0.98-1.06)$ & $1.05(1.02-1.08)$ \\
\hline \multicolumn{5}{|l|}{ Residence } \\
\hline Urban & $1.16(1.06-1.28)$ & $1.13(1.08-1.19)$ & $1.08(1.03-1.13)$ & $1.11(1.08-1.15)$ \\
\hline \multicolumn{5}{|l|}{ Zone } \\
\hline North Central & $1.48(1.26-1.74)$ & $2.15(1.96-2.35)$ & $1.59(1.47-1.72)$ & $1.76(1.67-1.86)$ \\
\hline North East & $1.17(1.01-1.35)$ & $1.81(1.67-1.96)$ & $1.63(1.52-1.74)$ & $1.60(1.53-1.68)$ \\
\hline North West & Reference & & & \\
\hline South East & $1.5(1.15-1.95)$ & $1.91(1.66-2.21)$ & $1.64(1.45-1.86)$ & $1.69(1.55-1.85)$ \\
\hline South South & $1.11(0.91-1.35)$ & $1.54(|.39-1.7|)$ & $0.96(0.88-1.05)$ & $1.16(1.08-1.23)$ \\
\hline South West & $1.56(1.26-1.95)$ & $1.87(1.66-2.1)$ & $1.36(1.24-1.50)$ & $1.54(1.44-1.65)$ \\
\hline \multicolumn{5}{|c|}{ Distance to health facility is big problem } \\
\hline Big problem & $1.23(1.10-1.38)$ & $1.21(1.15-1.26)$ & $1.44(1.38-1.5 \mid)$ & $1.31(1.27-1.35)$ \\
\hline \multicolumn{5}{|l|}{ Working } \\
\hline Yes & $1.21(1.10-1.33)$ & I.II (I.06-I.I7) & $1.19(1.14-1.24)$ & $1.16(1.13-1.20)$ \\
\hline \multicolumn{5}{|l|}{ Wealth quintile } \\
\hline Poorest & Reference & & & \\
\hline Middle & $1.49(1.32-1.68)$ & $1.67(1.57-1.77)$ & $1.55(1.47-1.64)$ & $1.57(1.52-1.64)$ \\
\hline Richest & $1.76(1.56-1.99)$ & $1.86(1.74-1.98)$ & $1.64(1.54-1.74)$ & I.7I (I.64-I.78) \\
\hline \multicolumn{5}{|c|}{ Wanted the pregnancy } \\
\hline Wanted then & Reference & & & \\
\hline Wanted later & $0.98(0.86-1.11)$ & $0.97(0.89-1.05)$ & $1.04(0.97-1.11)$ & $0.99(0.95-1.04)$ \\
\hline Not at all & $0.99(0.83-1.17)$ & $0.86(0.78-0.95)$ & $0.96(0.86-1.06)$ & $0.93(0.87-0.99)$ \\
\hline \multicolumn{5}{|c|}{ Previous pregnancies } \\
\hline None & Reference & & & \\
\hline 1 & $1.34(1.16-1.55)$ & $1.28(1.19-1.37)$ & $1.26(1.18-1.35)$ & $1.27(1.22-1.33)$ \\
\hline $2-3$ & $1.18(1.01-1.36)$ & $1.19(1.11-1.28)$ & $1.16(1.09-1.24)$ & $1.17(1.12-1.23)$ \\
\hline $4-6$ & $1.07(0.90-1.26)$ & $1.21(1 . \mid 1-1.31)$ & $1.14(1.06-1.23)$ & 1.15 (1.09-1.22) \\
\hline $7+$ & $1.22(0.99-1.50)$ & $1.13(1.01-1.25)$ & $1.04(0.95-1.15)$ & $1.10(1.03-1.17)$ \\
\hline
\end{tabular}

Abbreviations: ANC, antenatal care; DHS, Demographic and Health Survey; LWSP, living with sexual partner.

\section{Results}

A total of 45,690 pregnancies that were included in the analysis were those that either accessed ANC before the pregnancy came to an end or the pregnancies that came to an end as a result of delivery, termination, or abortion without a prior ANC visit. Nearly half (48.6\%) of these pregnancies were in 2013 survey, while 9.1\% were from the 2003 survey. Over two-thirds $(70 \%)$ of the women are rural dwellers, mostly (45\%) aged 25-34 years, and 47.1\% had no formal education, while only $37.9 \%$ were involved in the use of health care facility as shown in Table 1.

Overall, the prevalence of ANC use was $62.6 \%$. The prevalence fluctuated during the studied periods with the lowest prevalence of $60.5 \%$ in 2008 . The estimates in 2003 and 2013 were similar at $65.8 \%$ (Table 2 and Figure 1).

A further explorative analysis showed that the cumulative percentage for uptaking ANC services at each gestational age was higher in 2013 and least in 2008, as shown in Figure 2. The figure further depicts that enrolment into ANC services picked up at fourth and fifth months of gestation in each of the survey years.

The analysis of proportion of women who uptook ANC services showed that education, religion, tribe, involvement of women in decisions regarding their use of health care facilities, residence, zone of residence, and wealth status seemed to have clearly shown differences in ANC usage among the pregnant women (Table 2). For instance, women with higher education had a prevalence of $98.5 \%$ compared to those with no formal education at 37.7\%, 83.6\% among Christians compared with $48.4 \%$ among Islam's faithful, 96.0\% among the Igbos, 95.8\% among Yoruba compared with 37.1\% among the Hausa/Fulani. Other remarkable wide gaps were found among women involved in health care use decisions at $78.3 \%$ versus $52.4 \%$ among women who were not involved. Also, skilled 


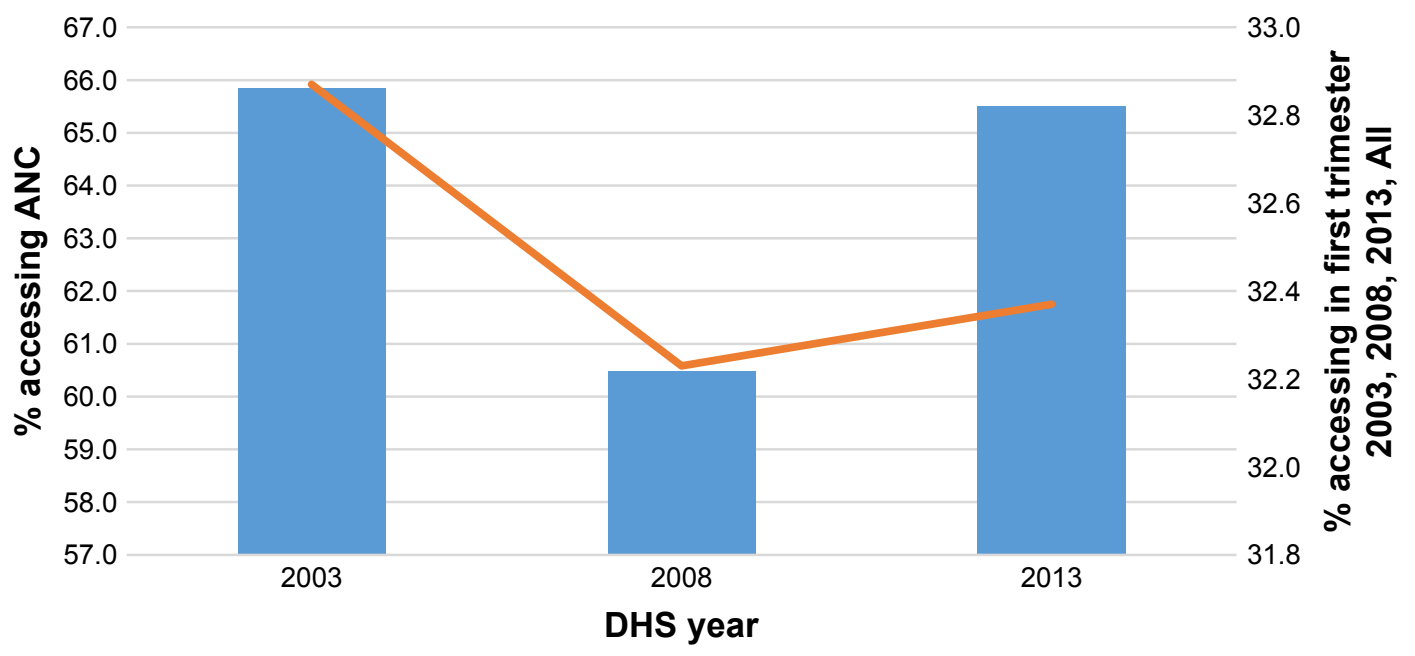

Accessed ANC $\longrightarrow$ Started in first trimester

Figure I Proportion attending ANC and proportion who started in the first trimester by survey years.

Abbreviations: ANC, antenatal care; DHS, Demographic and Health Survey.

birth attendants (SBA) use in urban area was $87.1 \%$ versus $52.4 \%$ in rural areas, $34.4 \%$ in North West compared with 94.1\% in South West, and $91 \%$ among women from the richest households against $39.5 \%$ in the poorest households. A test of association between survey years and ANC prevalence showed that association existed between the survey years and various categories of women except among those with higher education, never or formerly married, and women from richest households (Table 2). Further analysis showed that less than one-third (32.3\%) of the women accessed ANC within first trimester of pregnancy, with highest rate $(41.7 \%)$ among women with higher education and those from North Central (42.7\%).
The trimester of first ANC visit was significantly associated to all the characteristics studied except marital status, involvement in how women incomes are spent, and whether or not the pregnancy was wanted then. The number of previous pregnancies in the 2003 survey and marital status, involvement in how her incomes are spent, and whether or not the pregnancy was wanted then in 2013 were also significant.

The overall IR was 0.10 , that is, the probability of accessing ANC within a unit time ( 1 month) is $10 \%$. This translates to 100 per 1,000 person-year uptaking ANC provided that the pregnant women had not attended ANC before that time. This varied across the survey years and other women

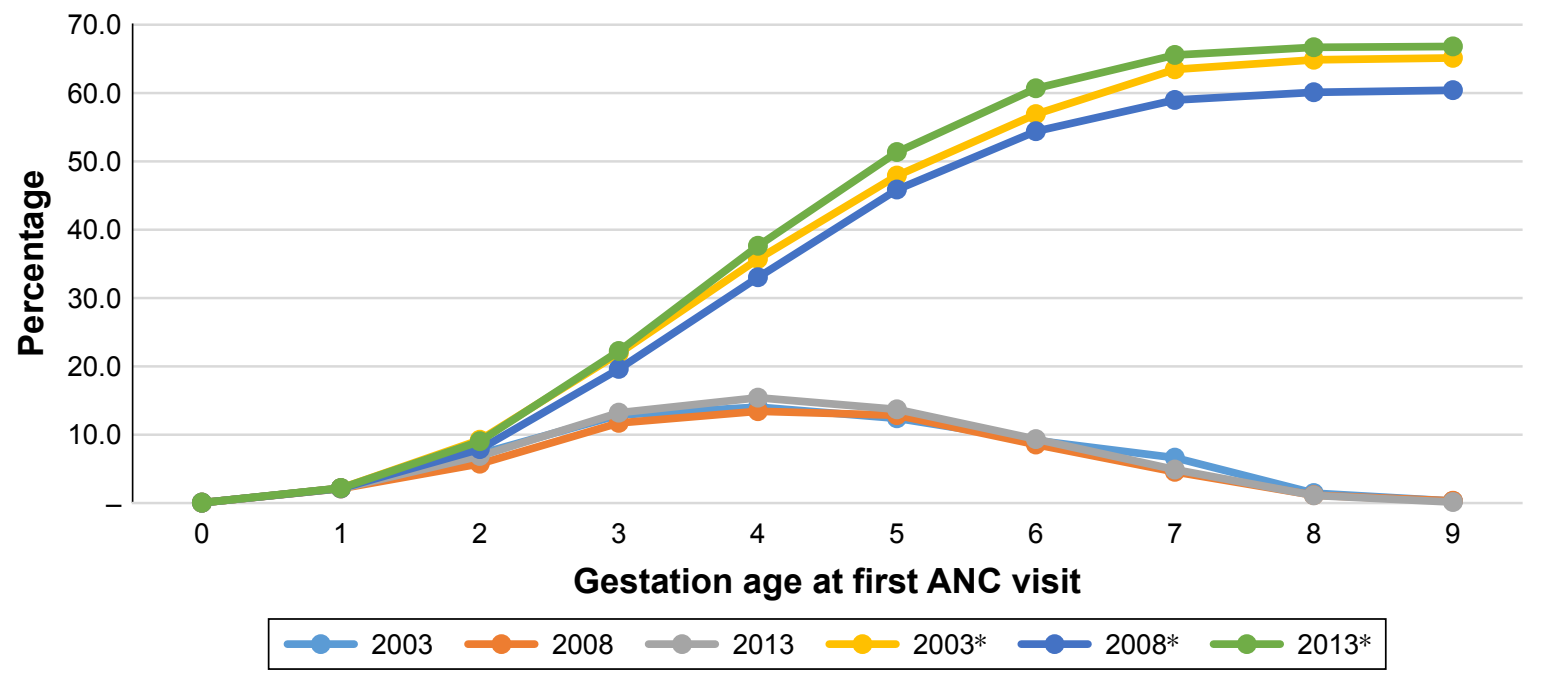

Figure 2 Percentage and cumulative percentage distribution of the month of ANC initiation by survey years. Note: *Cumulative probability distribution.

Abbreviation: ANC, antenatal care. 

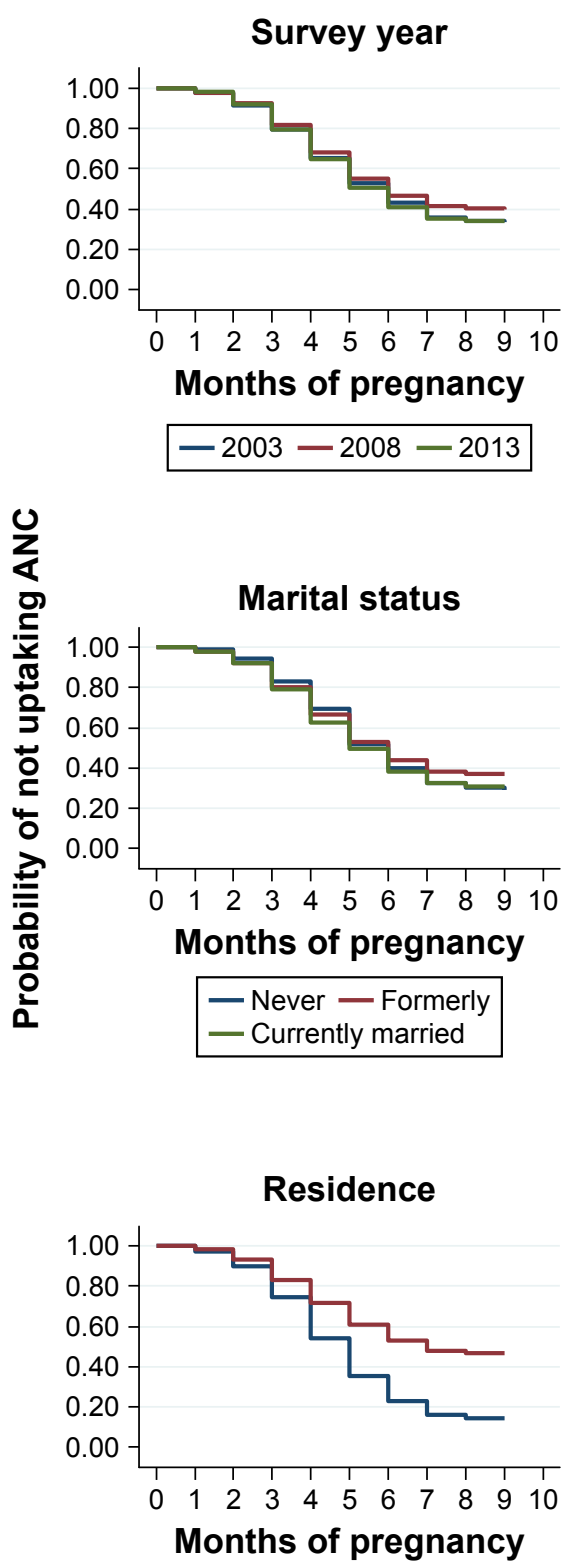

- Urban - Rural
Maternal age

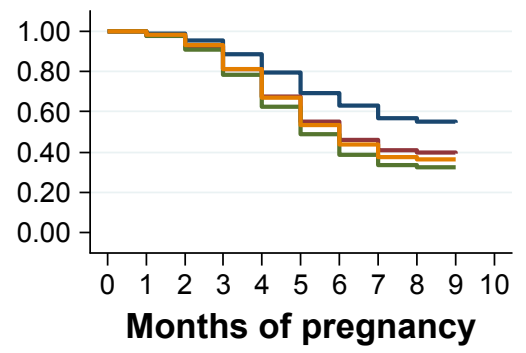

$\begin{array}{ll}-15-19 \mathrm{yr} & -20-24 \mathrm{yr} \\ -25-34 \mathrm{yr} & -35+\mathrm{yr}\end{array}$

Involved in health facility use decision
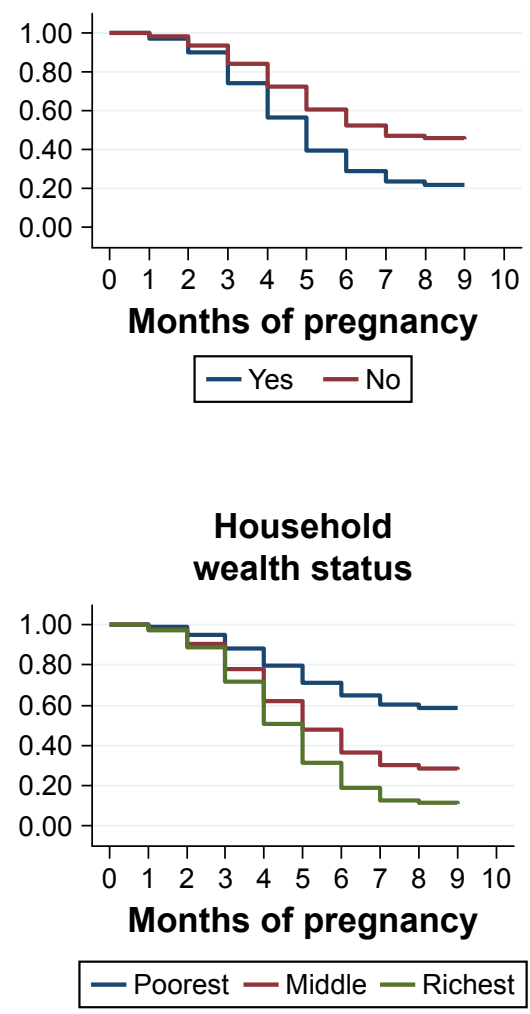

Educational attainment
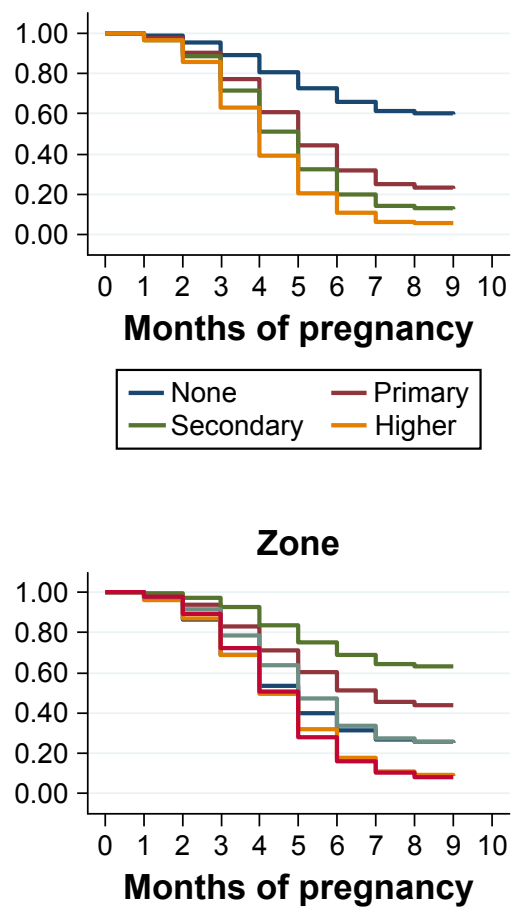

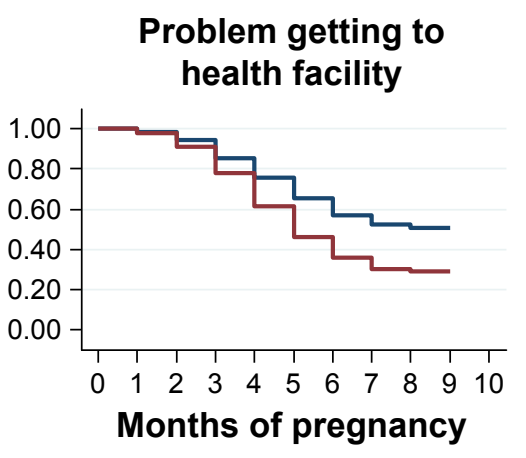

- Big problem — Not big problem

Figure 3 Survival functions of first ANC visit by survey years and respondent characteristics. Abbreviation: ANC, antenatal care.

characteristics. However, the median survival time was similar across the survey years at 6 months, as shown in Table 3 and Figure 3. The highest probabilities of uptaking ANC per month was found among women from richest households (0.18), South West and South East women (0.19), Yoruba (0.20), Igbo (0.19), and women with higher education (0.21). These are the categories of women having the smallest median survival time. The longest median survival time was observed among Muslims and women with over 6 previous pregnancies at 8 months each.

The log-rank test of equality of survival functions showed that all the survival functions of the categories for each characteristic studied were significantly different (Figure 3 and Table 3), except for marital status and involvement of women in decisions on how her earnings are spent.

The HR for each characteristic in the independent Cox proportional hazard regression model showed that all the characteristics significantly determined the timing of first ANC visit except involvement of women in decisions on how her earnings are spent (not shown in the tables). For survey year 2003, after controlling for other variables, women aged 35-49 years had significant higher hazard (18\%) of accessing ANC compared with women aged $15-19$ years $(\mathrm{aHR}=1.18$, 95\% CI: 0.94-1.48). Moreover, the hazard of accessing ANC 
for the first time was 56\% times higher in South West than in North West (aHR $=1.56,95 \%$ CI: $1.26-1.95)$. In comparison, maternal age, education, tribe, rural/urban residence, zone of residence, whether distance to health care facility was a big problem, wealth quintile, working status, and number of pregnancies before the index pregnancy were significant determinants in each of the survey years 2003, 2008, and 2013. However, religion that significantly determined ANC timing in 2003 and 2008 was no longer significant in 2013. Furthermore, involvement in decisions on her use of health care facilities was significant only in 2008. The last panel of Table 4 shows the aHRs when the survey years were included in the model. The hazard of the timing of first ANC visit was higher in the years 2003 and 2013 than in 2008 (aHR $=1.19$, 95\% CI: $1.12-1.26$ and aHR $=1.08,95 \%$ CI: $1.06-1.11$, respectively). Besides the desirability of the pregnancy, all other women characteristics significantly determined the timing of first ANC visit. Higher hazards were found among older women, those with better education, being a Yoruba or Igbo, involved in women health care use, residing in urban areas and in southern regions, having no big problem accessing health facilities, working currently, from household in richest quintiles, and with lower gravidity.

\section{Discussion}

The main aim of the study was to assess the trends in the timing of first visits to ANC service based on the data from three most recent NDHS surveys. The surveys were conducted in 2003, 2008, and 2013. The study revealed some fluctuations in ANC utilization with 2008 having the lowest and 2003 and 2013 similar prevalence rates. The $6 \%$ drop in ANC utilization in 2008 compared to the prevalence obtained in 2003 and 2013 may be linked to growth in the number of community health officers and traditional birth attendants in Nigeria in mid-2000. Most stakeholders have discouraged the use of these nonskilled professionals thereafter. While timings of first ANC visits varied across the years, women characteristics significantly influenced the timings. Particularly, women's level of education, religion, tribe, involvement of women in decisions regarding their use of health care facilities, residence, zone of residence, and wealth status determined when they started ANC visit. The overall chance of uptaking ANC services by the women per unit time (month) is $10 \%$, which varied across the survey years and other woman characteristics. However, median survival times were similar across the survey years. In 2013, the variability of the likelihood of women to uptake ANC returned to the level it was in 2003.

Although WHO recommends that the number of ANC visits is important, the timing of the first visit is also equally important. ${ }^{22} \mathrm{We}$ analyzed the timing of the first visit and found that less than one-third of the pregnant women made first ANC visit within the first trimester of pregnancy. Also, the overall median time was 6 months. These statistics are generally higher than the global average and lower when compared to the recent findings of average of 5 gestational months before first ANC visit in Tanzania. ${ }^{30}$ Late initiation of ANC by Uganda women has also been documented. ${ }^{31}$ However, earlier initiation of ANC visit has been reported in Ethiopia where 35.4\% of pregnant women started ANC in the first trimester and average initiation time of 4.5 months. ${ }^{32}$ Late initiation of ANC could adversely affect the outcome of pregnancies. This may not be disconnected from poor maternal and child health in Nigeria. Stakeholders should work more on encouraging and providing an enabling environment for pregnant women to start ANC visit earlier.

The highest IR of first ANC visit was found among women from richest households, in urban areas, living in South West and South East, and those with higher education. These results are in line with earlier results. ${ }^{17}$ The current study found pregnant women living in urban areas start ANC visit earlier than their contemporaries in rural areas. Similar assertions were made earlier in Tanzania, wherein earlier initiation were found to be more common in Addis Ababa than in the hinterlands. ${ }^{32}$ This could be due to better presence of ANC providers in urban areas than in rural areas. Also, distances to health facilities are generally shorter in urban centers. There is need to make ANC facilities more accessible to all. We found earlier initiation of ANC visit in the southern regions than in the northern regions except in the North Central. Educational attainment might have played a vital role here as the northern zones have been reported to have highest illiteracy levels in the country. ${ }^{15,33,34}$ In the same vein, we found women with higher education to have the higher likelihood of earlier initiation of ANC visits. Similar findings have been documented. ${ }^{17,27,35}$ Women, especially the girl child, should be educated so as to be prepared for such eventualities such as ANC attendance in the future.

Furthermore, higher education leads to better jobs which yield higher income. Pregnant women from households in richer wealth quintiles had higher likelihood to book ANC services early compared to those from households in poorer wealth categories. Several studies have associated poorer financial status to increased odds of underutilizing ANC services among the pregnant women. These results are consistent with those reported in the literature. ${ }^{21,30-32}$ For instance, a recent Nigerian study found that the woman in the wealthiest quintile had a higher likelihood of adequately utilizing ANC and that the chances of using ANC increases with the level of wealth. ${ }^{21}$ 
There were differences in timing of first ANC visit along religion and ethnicity divides. This could be as a result of the natural arrangement of Nigerian settlements as the North is dominated by Muslims and the South is dominated by Christians and other religions. Yoruba or Igbo women, who reside more in the South, had higher tendencies to practice Christianity and are more likely to start ANC earlier. This result is also in line with the existing report that most husbands practicing Islam forbid their wives from seeking health services without outright permission. ${ }^{34}$ Same reason could be ascribed to delayed initiation of ANC among women who are not involved in their health care decisions and in how their incomes are spent, although women involvement in decisions on their usage of health care facilities was significant only in 2008.

The outcome of the Cox proportional hazard regression model used to model the determinants of the timing of first ANC visit showed that all characteristics considered significantly determined the timing of first ANC visit across the years except for involvement of women in decision on how their incomes are spent. A recent Nigerian study had also identified a strong positive relationship between the utilization of ANC services and age of mother, place of residence (rural/urban), mother's and husband's level of education, working status of the woman, household wealth quintile, health insurance enrolment, religion, and woman's decision-making autonomy. ${ }^{36}$ They further highlighted that the influence of maternal age on the use of ANC is unclear and inconsistent. In our study, women aged 35-49 years had significant higher hazard of accessing ANC in 2003 and 2013 compared with women aged 15-19 years, while in 2008 women aged 25-34 years had a higher hazard. The findings of our study are also similar to a study of 15 developing countries which found that younger women were less likely to use ANC services. ${ }^{37}$

\section{Conclusion}

Initiation of ANC visit in Nigeria is generally late with most women making first visit during sixth month of gestation, although few variations existed across the years studied. An increase in the coverage of ANC visits recorded between 2003 and 2013 was not accompanied by earlier commencement of ANC visit during the same period. The hazard of the timing of first visit to ANC facilities was higher in 2003 and 2013 than in 2008. Higher hazard of ANC initiation was found among older women, those with better education, being a Yoruba or Igbo, involved in women health care use, residing in urban areas and in southern regions, having no problem with accessing health facilities, working currently, from households in richest quintiles, and having fewer previous pregnancies. Late initiation of ANC could worsen pregnancy outcomes as necessary timely intervention to salvage life of mother and child would be delayed or totally too late as a result of late visits. The worst affected women are the rural dwellers, women with little or no education, those with weak decision-making power, and those from households in poorer wealth quintiles. The determinants of first ANC visit did not vary much between 2003 and 2013. Stakeholders should do more at ensuring that all pregnant women start ANC visit earlier.

\section{Study limitations}

A secondary data was used for this study; we therefore did not have full control on the choices of independent variables. The data was self-reported. We have relied on these preexisting data which we were unable to verify independently and this might have introduced recall bias. However, the large sample size and the national representativeness of the data would have neutralized the likely effect of such bias.

\section{Acknowledgments}

We acknowledge ICF Macro and the National Population Council on behalf of the Federal Government of Nigeria for granting us access to the data used for this study.

\section{Disclosure}

The authors report no conflicts of interest in this work.

\section{References}

1. Saronga HP, Duysburgh E, Massawe S, et al. Efficiency of antenatal care and childbirth services in selected primary health care facilities in rural Tanzania: a cross-sectional study. BMC Health Serv Res. 2014; 14(1):1-11.

2. United Nations. Sustainable Development Goals (SDG). Washington, DC; 2015. Available from: http://www.un.org/sustainabledevelopment/ sustainable-development-goals/. Accessed March 18, 2017.

3. Simkhada B, Teijlingen E, Porter M, Simkhada P. Factors affecting the utilization of antenatal care in developing countries: systematic review of the literature. J Adv Nurs. 2008;61(3):244-260.

4. Lincetto O, Mothebesoane-anoh S, Gomez P, Munjanja S. Antenatal Care: Opportunities for Africa's Newborns. New York; 2010. Available from: http://www.who.int/pmnch/media/publications/aonsectionIII_2.pdf. Accessed March 15, 2017.

5. WHO. Mother-Baby Package: Implementing Safe Motherhood in Countries. Geneva: World Health Organization; 1994:114.

6. Berhan Y, Berhan A. Antenatal care as a means of increasing birth in the health facility and reducing maternal mortality: a systematic review. Ethiop J Health Sci. 2014;24(0 Supp1):93-104.

7. Villar JEA. WHO antenatal care randomized trial for the evaluation of a new model of routine antenatal care. Lancet. 2001;357:1565-1570.

8. Chen X-K, Wen SW, Yang Q, Walker MC. Adequacy of prenatal care and neonatal mortality in infants born to mothers with and without antenatal high-risk conditions. Aust N Z J Obstet Gynaecol. 2007;47(2): $122-127$. 
9. McDonagh M. Is antenatal care effective in reducing maternal morbidity and mortality? Health Policy Plan. 1996;11(1):1-15.

10. Fagbamigbe AF, Idemudia ES. Assessment of quality of antenatal care services in Nigeria: evidence from a population-based survey. Reprod Health. 2015;12(8):1-9.

11. Babalola S, Fatusi AO. Determinants of use of maternal health services in Nigeria: looking beyond individual and household factors. $B M C$ Pregnancy Childbirth. 2009;9(43):1-15.

12. Osungbade K, Shaahu VN, Uchendu OC. Clinical audit of antenatal service provision in Nigeria. Health Care Women Int. 2011;32(5): 441-452.

13. WHO. World Health Statistics 2007: WHO, Geneva, Switzerland

14. Fagbamigbe AF, Idemudia ES. Barriers to antenatal care use in Nigeria: evidences from non-users and implications for maternal health programming. BMC Pregnancy Childbirth. 2015;15(95):1-10.

15. National Population Commission (Nigeria) and ICF International. Nigeria Demographic and Health Survey 2013. Abuja, Nigeria: NPC and ICF International; 2014.

16. WHO. World Health Statistics, 2015. Washington, DC; 2015. Available from: http://apps.who.int/iris/bitstream/10665/170250/1/97892406944 39_eng.pdf. Accessed March 15, 2017.

17. Federal Ministry of Health Nigeria. National HIV/AIDS and Reproductive Health and Serological Survey, 2012 (NARHS Plus). Abuja, Nigeria: Federal Ministry of Health; 2013.

18. Russo G, Herrin AN, Pons MC. Household health care facility utilization in the Philippines. Asia Pac J Public Health. 1996;9:6-12.

19. Mekonnen Y, Mekonnen A. Utilization of Maternal Health Care Services in Ethiopia. Calverton, MA: ORC Macro; 2002:1-23.

20. Adekunle AO, Arowojolu AO, Adedimeji AA, Okunlola MA. Emergency contraception: survey of knowledge, attitudes and practice of healthcare professionals in Ibadan. $J$ Obstet Gynecol. 2000; 20(3):284-289.

21. Fagbamigbe AF, Idemudia ES. Wealth and antenatal care utilization in Nigeria: policy implications. Health Care Women Int. 2016;38(1): 17-37.

22. Mallick L, Dahiru T, MacQuarrie LDK. Trends in Maternal Health in Nigeria, 2003-2013. Vol 102. Rockville, MA: ICF International; 2016.

23. National Population Commission (Nigeria) and ICF International Nigeria Demographic and Health Survey, 2008. Abuja, Nigeria: DHS Measure Macro (NY), Nigeria Population Commission; 2009.

24. Fagbamigbe AF, Idemudia ES. Survival analysis and determinants of timing of first birth after marriage in Nigeria. Afr Popul Stud. 2016; 30(2):2444-2457.
25. Kyei N, Campbell OMR, Gabrysch S. The influence of distance and level of service provision on antenatal care use in rural Zambia. PLoS One. 2012;7(10):1-6.

26. Gabrysch S, Campbell OMR. Still too far to walk: literature review of the determinants of delivery service use. BMC Pregnancy Childbirth. 2009;9(1-9):34.

27. Arthur E. Wealth and antenatal care use: implications for maternal health care utilisation in Ghana. Health Econ Rev. 2012;2:14(14):1-18.

28. Adebowale AS, Fagbamigbe AF, Okareh TO, Lawal GO. Survival analysis of timing of first marriage among women of reproductive age in Nigeria: regional differences. Afr J Reprod Health. 2012;16(4): 95-107.

29. Fagbamigbe AF, Idemudia ES. Survival analysis and prognostic factors of timing of first childbirth among women in Nigeria. BMC Pregnancy Childbirth. 2016;16(102):1-12.

30. Gross K, Alba S, Glass TR, et al. Timing of antenatal care for adolescent and adult pregnant women in south-eastern Tanzania. BMC Pregnancy Childbirth. 2012;12(1):16.

31. Kisuule I, Kaye DK, Najjuka F, et al. Timing and reasons for coming late for the first antenatal care visit by pregnant women at Mulago hospital, Kampala Uganda. BMC Pregnancy Childbirth. 2013;13(1):121.

32. Gudayu TW, Woldeyohannes SM, Abdo AA. Timing and factors associated with first antenatal care booking among pregnant mothers in Gondar Town, North West Ethiopia. BMC Pregnancy Childbirth. 2014; 14(1):287.

33. Ashir GM, Doctor HV, Afenyadu GY. Performance based financing and uptake of maternal and child health services in Yobe Sate, Northern Nigeria. Glob J Health Sci. 2013;5(3):34-41.

34. Doctor H, Bairagi R, Findley SE, Helleringer S. Northern Nigeria Maternal, Newborn and Child Health Programme: selected analyses from population-based baseline survey. Open Demogr J. 2011; $4: 11-21$

35. Titaley CR, Dibley MJ, Roberts CL. Factors associated with underutilization of antenatal care services in Indonesia: results of Indonesia Demographic and Health Survey 2002/2003 and 2007. BMC Public Health. 2010;10(485):1-10.

36. Dahiru T, Oche OM. Determinants of antenatal care, institutional delivery and postnatal care services utilization in Nigeria. Pan Afr Med J. 2015;21:321.

37. Reynolds BHW, Wong EL, Reynolds HW. Adolescents' use of maternal and child health services in developing countries. Int Fam Plan Perspect. 2006;32(1):6-16.
International Journal of Women's Health

\section{Publish your work in this journal}

The International Journal of Women's Health is an international, peerreviewed open-access journal publishing original research, reports, editorials, reviews and commentaries on all aspects of women's healthcare including gynecology, obstetrics, and breast cancer. The manuscript management system is completely online and includes

\section{Dovepress}

a very quick and fair peer-review system, which is all easy to use. Visit http://www.dovepress.com/testimonials.php to read real quotes from published authors. 